\title{
Comparison Between Mass Attenuation Coefficient of Metals (Fe, Ag, Sn, Pt, Au, Pb) According to Their Atomic Number
}

\author{
Viyan Jamal Jalal ${ }^{1, *}$, Bawar Mohammed Faraj ${ }^{2}$ iD, Sarkew S. Abdulkareem ${ }^{1}$ iD \\ ${ }^{1}$ Department of Physics, College of Science, University of Halabja, Halabja, 46018, Iraq \\ ${ }^{2}$ Computer Science Department, College of Science, University of Halabja, Halabja, 46018, Iraq \\ *Corresponding Author: Viyan Jamal Jalal, E-mail: vian.jalal@uoh.edu.iq
}

\begin{abstract}
Article Info
Abstract

Article History

The paper describes the X-ray attenuation in materials according to their atomic number, using

Received Aug 1, 2021 six different materials from low atomic number to higher atomic number, Iron (Fe-26) as low

Revised Aug 26, 2021 atomic number, silver (Ag-47), tin ( $\mathrm{Sn}-50)$, platinum (Pt-78), gold (Au-79) and lead (Pb-82)

Accepted Aug 28, 2021 as high atomic number. Using the data were taken from the NIST Standard Reference

\section{Keywords}

X-ray attenuation Database 126. From the J. H. Hubbell and S. M. Seltzer work, a table of contents was used.

Mass attenuation coefficient Whereas The mass attenuation coefficient for the above six elements was taken and compared
\end{abstract}

$\mathrm{X}$-ray transmission

Atomic number

Copyright: @ 2021 EngiSciece Publisher All rights reserved

\section{Introduction}

When an X-ray beam incident on an atomic target, two processes may occur; the beam may be absorbed (attenuated) with an ejection of electrons from the atoms, or the beam may be scattered.[1,2] Attenuation is defined as an intensity loss of incident X-rays passing through a matter. The intensity, I0, of a monochromatic beam (X-ray) decreases exponentially:

$$
\mathrm{I}=\mathrm{I}_{0} \mathrm{e}^{-\mu \mathrm{x}}
$$

where the linear absorption coefficient, $\mu(\mathrm{cm})^{-1}$ represents the average number of absorption and scattering processes that a single photon undergoes through an absorber of thickness $\mathrm{x}(\mathrm{cm}) .[3]$ the mass attenuation coefficient is equal to the linear attenuation coefficient to the density of the element $\mu / \rho\left(\mathrm{cm}^{2} / \mathrm{g}\right)$.

Many experimental measurements to determine the attenuation coefficients in solid materials have been presented $[4,5]$. The $[6,7,8]$ describe the attenuation coefficient concerning energy range values. And [9] measures the mass attenuation coefficient for ( $\mathrm{Na}, \mathrm{Mg}, \mathrm{Al}, \mathrm{Ca}$, and $\mathrm{Fe}$ ).

The present work is to compare the mass attenuation coefficient for six different elements, at the same $\mathrm{X}$-ray energy value, concerning their atomic numbers 


\section{Method}

NIST X-ray Attenuation Databases[10] were used in work [1], and we used their results in the table of their data to compare the mass attenuation coefficient of the elements Iron (Fe-26) as low atomic number, silver (Ag-47), tin (Sn-50), platinum (Pt-78), gold (Au-79) and lead (Pb-82) as high atomic number, at the same energy value of X-ray $30 \mathrm{KeV}$. From table 3 of the X-Ray Mass Attenuation Coefficients[1], we get the following data.

\section{Data}

Table 1. Mass attenuation coefficients for metals with different atomic number

\begin{tabular}{llll}
\hline Elements & $\mathbf{Z}$ & X-ray Energy $(\mathbf{K e V})$ & $\boldsymbol{\mu}(\mathbf{c m} 2 / \mathbf{g})$ \\
\hline $\mathrm{Fe}$ & 26 & 30 & 8.176 \\
$\mathrm{Ag}$ & 47 & 30 & 36.68 \\
$\mathrm{Sn}$ & 50 & 30 & 41.21 \\
$\mathrm{Pt}$ & 78 & 30 & 26.41 \\
$\mathrm{Au}$ & 79 & 30 & 27.52 \\
$\mathrm{~Pb}$ & 82 & 30 & 30.32 \\
\hline
\end{tabular}

\section{Discussion}

The six elements shown in the above table are chosen to compare the mass attenuation coefficient as a function of the atomic number contained in the medium and high range of atomic number. The attenuation coefficient is directly proportional to the atomic and electronic cross section [11-13], and the atomic crosssection is proportional to the atomic number[9], so there can be said that the attenuation coefficient is proportional to the atomic number of the elements (materials). But if looking at the results shown in table 1, one can see that this was true if compared between Fe, $\mathrm{Ag}$, and $\mathrm{Sn}$, and it was false between $\mathrm{Sn}$ and the three other elements $\mathrm{Pt}, \mathrm{Au}$, and $\mathrm{Pb}$. The attenuation coefficient for $\mathrm{Sn}$ is more significant than that for $\mathrm{Fe}$ and $\mathrm{Ag}$, while the atomic number of $\mathrm{Sn}$ is greater than the atomic number for Fe and $\mathrm{Ag}$. Still, for Pt, $\mathrm{Au}$, and $\mathrm{Pb}$ which have a more significant atomic number than $\mathrm{Sn}$, the attenuation coefficient is less than for $\mathrm{Sn}$. If the comparison will be taken only between the higher atomic number elements $\mathrm{Pt}, \mathrm{Au}$ and $\mathrm{Pb}$, then 
one can say that the mass attenuation coefficient is proportional to the atomic number of the element, but this comparision will not be true if taken between these three elements ( $\mathrm{Pt}, \mathrm{Au}$, and $\mathrm{Pb}$ ) and $\mathrm{Ag}$, and $\mathrm{Sn}$.

\section{Conclusion}

X-ray transmission in metals depends on the atomic number of the elements (metals), and the mass attenuation coefficient for six different metals (Fe-26, Ag-47, Sn-50, Pt-78, Au-79, and Pb-82) was compared. The attenuation coefficient is proportional to the atomic number; the data agreed with the first three elements and the second three elements separately, but disagreed if the comparison takes place between $\mathrm{Ag}$ and the $\mathrm{Pt}, \mathrm{Au}$, and $\mathrm{Pb}$ or between these three metals and $\mathrm{Sn}$. For example, the atomic number of $\mathrm{Pb}$ is larger than the atomic number of $\mathrm{Sn}$, the attenuation coefficient for $\mathrm{Pb}$ is lesser than that for $\mathrm{Sn}$. This comparison will have to take attention to it and is needed to describe the cause of these differences.

Declaration of Competing Interest, The authors declare no conflict of interest.

\section{References}

[1] Seltzer, J. H. H. and S. M., 1996. X-Ray Mass Attenuation Coefficients. NIST, U.S. Secretary of Commerce on behalf of the United States of America. Available at: https://www.nist.gov/pml/x-ray-mass-attenuation-coefficients (Accessed: 26 Apr 2021).

[2] Warren, B.E., 1990. X-ray Diffraction. Courier Corporation, New York, USA.

[3] Sasaki, S., 1990. X-ray absorption coefficients of the elements (Li to Bi, U) (No. KEK--90-16). National Lab. for High Energy Physics.

[4] Abdullah, K.K., Ramachandran, N., Nair, K.K., Babu, B.R.S., Joseph, A., Thomas, R. and Varier, K.M., 2008. Attenuation studies near K-absorption edges using Compton scattered 241 Am gamma rays. Pramana, 70(4), pp.633-641.

[5] Saloman, E.B., Hubbell, J.H. and Scofield, J.H., 1988. X-ray attenuation cross sections for energies $100 \mathrm{eV}$ to $100 \mathrm{keV}$ and elements $Z=1$ to $Z=92$. Atomic Data and Nuclear Data Tables, 38(1), pp.1-196.

[6] Cheewasukhanont, W., Limkitjaroenporn, P. and Kaewkhao, J., 2020, March. Calculation of The Radiation Shielding Parameters in Long Ranges of Photon Energy: Bismuth Sodium Borate Glass. In Journal of Physics: Conference Series (Vol. 1485, No. 1, p. 012027). IOP Publishing.

[7] Gerward, L., 1982. X-Ray attenuation coefficients for copper in the energy range 5 to 50 ke V. Zeitschrift für Naturforschung A, 37(5), pp.451-459.

[8] Turgut, Ü., Büyükkasap, E., Şimşek, Ö., Ertuğrul, M. and Doğan, O., 1998. Determination of X-Ray Total Attenuation Coefficient in Zr, Ag, In for Energy Range Between 10.5-111.9 keV. Acta Physica Polonica A, 5(93), pp.693-700.

[9] Akça, B. and Erzeneoğlu, S.Z., 2014. The mass attenuation coefficients, electronic, atomic, and molecular cross sections, effective atomic numbers, and electron densities for compounds of some biomedically important elements at $59.5 \mathrm{keV}$. Science and Technology of Nuclear Installations, 2014.

[10] NIST, 2016. Note on NIST X-ray Attenuation Databases. Available at: https://www.nist.gov/pml/note-nist-X-rayattenuation-databases. (Accessed: 26 Aug 2021). 
[11] Jackson, D.F. and Hawkes, D.J., 1981. X-ray attenuation coefficients of elements and mixtures. Physics Reports, 70(3), pp.169-233.

[12] Singh, K. and Gerward, L., 2002. Summary of existing information on gamma-ray and X-ray attenuation coefficients of solutions. Indian Journal of Pure and Applied Physics 40(9), pp: 643-649.

[13] Manohara, S.R., Hanagodimath, S.M. and Gerward, L., 2008. Energy dependence of effective atomic numbers for photon energy absorption and photon interaction: studies of some biological molecules in the energy range $1 \mathrm{keV}-20 \mathrm{MeV}$. Medical physics, 35(1), pp.388-402. 